\title{
Multi-agent system with information fusion for intelligent lighting control
}

\author{
Liangzhou Wang, Weihong Yu and Jony Hock \\ Transportation Management College \\ University of Dalian Maritime \\ Dalian, Liaoning Province, China \\ qjyzwlz@126.com
}

\begin{abstract}
Intelligent lighting system is the core component of the smart home. In order to achieve user-centric intelligent control, improve indoor visual comfort level and saving energy at the same time, we have designed a multi-agent intelligent lighting system based on multi-source information fusion. Multi-agent systems able to improve the interactivity of the system, so that the subsystem concurrent processing of data and information for decision making distributions to achieve a closed-loop control. An intelligent data fusion model is proposed to fuse ambient light, occupancy, energy limitations, user preferences and other information for decision making. We use ANFIS modeling algorithm for environmental control of occupancy estimated. Finally, through FIS algorithm, we can implements the entire lighting control subsystem.
\end{abstract}

Keyword-intelligent lighting control, multi-agent, information fusion, ANFIS algorithm, smart home

\section{INTRODUCTION}

Intelligent lighting system is the core component of the smart home. A well-designed, precise intelligent lighting control systems not only meet daily lighting needs of the people, but also to be able to save energy and time, to achieve a variety of control purposes. With this intelligent lighting system, we not only control household equipment artificially with smart device, but also to manage variety of devices through intelligent decision-making system automatically by taking advantage of multi-source information fusion. For this purpose, the multi-agent technology with information fusion can be adopted to control the intelligent lighting system, and the control goal is to maintain the high-level comfort with the minimum power consumption in different operating conditions [1].

There has been much research on the intelligent lighting system. Some of the studies focus on automatic control with intelligent decision-making based on occupancy estimation and daylight-linked lighting[5-6]. But with the popularity of smart mobile devices, we prefer to control illumination pattern combined people's decision-making with intelligent person mobile device. In much research, multi-agent technology has been utilized to develop intelligent control systems for building's energy management. Dounis et al. [2] developed a fuzzy logic controller for the visual comfort. Dounis, RuiYang[3] develop a Multi-agent control system with information fusion based comfort model. This paper developed a novel multi-agent system model for intelligent building energy and comfort management based on occupant behaviors, but it does not establish a common information fusion model. In order to integrate the information from ambient environment and human pattern of behavior , combine automatic control with human control. In this paper, we design a multi-agent lighting control architecture based on android platform. Four type agents be arranged at different platform including android system and personal computer. The designed multi-agent system can be applied to enable the intelligent lighting system to interact with its users through a wireless network to for achieving the goal of user-centered control. Moreover, The multi-source information fusion model based on ANFIS algorithm has been designed to blend multi-source information such as ambient visual information, occupancy information, energy information and occupants' preference information to make decision for artificial control and automatic control. Finally, we use fuzzy inference toolbox in the MATLAB to simulate natural lighting.

\section{PRINCIPLES OF INTERACTION OF MULTI-AGENT SYSTEM FOR THE INTELLIGENT LIGHTING CONTROL}

Multi-agent control system composed of several agents. Although functionally each agent has a different function, each agent still has common characteristics. For example, each agency must have a certain degree of autonomy, each agent can communicate with each other by using their own language, and the most important that each agent can determine all of their own behavior in order to achieve the main goal[2] .

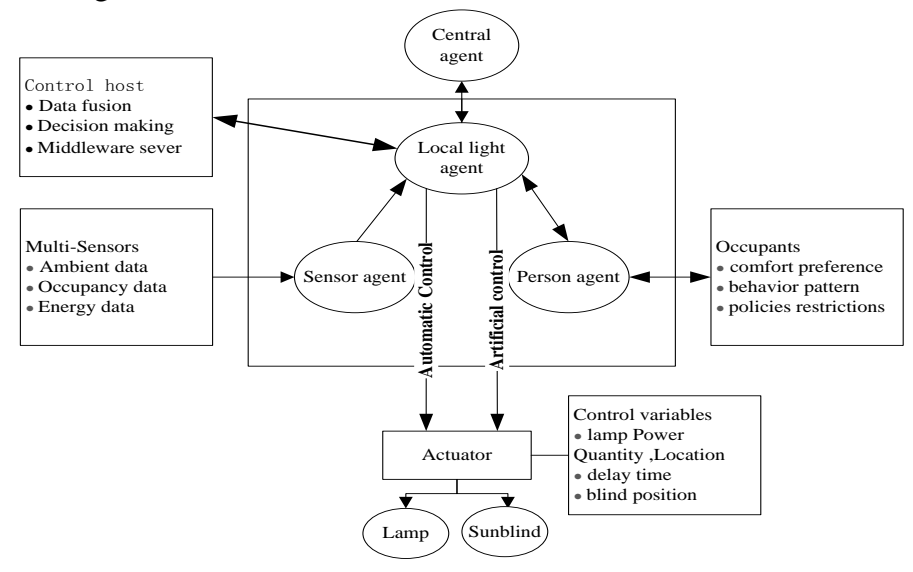

Fig. 1. Principles of interaction of multi-agent lighting system

Figure 1 describes how the smart lighting control system work in generally based on multi-agent system within the 
scope of the smart home. Multi-agent systems receive information from sensors and multi-user, after the multi-agent system make decisions that ultimately applied to the actuator control lighting. The sensors are distributed throughout the area in order to monitor the performance of smart appropriately and accurately.

In a multi-agent lighting system suggested four types of agents, central agent, local lighting agent, agent sensor and personal agent. In this multi-agent lighting system, personal agent is located in the user's control terminal. The personal agent communicates with local agents to provide information about occupant's preferences and command information. It also receives feedback from local agent concerning the environmental changes and the interactions between humans and their environment. In the smart home system, there are a variety of local agent, each agent is responsible for controlling a subsystem. Local lighting agents are designed to control each lighting subsystem []. Local agents coordinated by the central agent management. Central agent is responsible for the local agent configuration, communication, etc. Sensor agent is the intermediary sensor data collection, it receive the data through a wireless network from card which located in the intermediary sensor, and responsible for communication with the local agent to transmit the data to central server.

\section{A. Personal Agent}

In order to achieve the user-centered intelligent control.personal agency tasked to facilitate responding to the needs of occupant. It work as an agent's personal assistant who manages information that obtained from occupant, and then deliver the orders given by occupant, and presenting feedback to the occupant. Personal agent is located in the system control terminal devices such as personal Computer, smart phone, wearable device, etc. In one hand, personal agent can remember user's preferences through the intelligent algorithm for simulating the user behavior patterns. On the other hand, if the surrounding environment changes, or if the user changes has changed the lighting setups, personal agent will present feedback of local agent to the user, and the user's preferences and instructions transmitted to the control center, to realize human control. Personal agent terminal have diversity of control mode. People can set lighting scene model, for example, one button to select the desired lighting.

\section{B. Local Lighting Agent}

Local lighting agent in a multi-agent system has a very important role. In addition to responsibilities as a mediator and information provider, also has an important role in decision-making and executive control in a multi-agent system, local lighting agent is located in the control center of server, which receives sensor data sensor agent, then transfer data to the data fusion center for analysis and processing. Beside that, local lighting agent also interacts with the person agent to receive information and instructions. Local agent is a direct interface to the smart home backend server, database, control center and computing center.

\section{Sensor Agent}

Multiple sensor sensing the presence of a large number redundant of environmental data and directly influence the performance of the network communication. Sensor agent is proposed to carry out the preprocessing of the raw sensor data. Many of similar sensor data after its filtering and data level fusion, then will improve accuracy and reduced redundancy.

\section{Central Agent}

Central Agents have two major functions: agent system configuration and facilitating collaboration between local agents. Intelligent lighting system is a subsystem of smart home, there are lot of other subsystem which are directly manage by local agents. And central agent is responsible for the management of these local agents, configuration and coordination. Central agent allows administrators to enable or stop a local agent, add or delete an agent, it also provide a link to local agent platform to implement interaction of multiple local agents.

After processing the central controller information which comes from sensor agents and person agents, the decision information through the local agent distributed to the corresponding actuator. Variables that need to have direct control such as power of lamp (light intensity directly determined), number of capacity, position (the position that requires illumination which was estimated by the occupancy), blind open position ( $0 \%$ means close, $100 \%$ means open completely)

\section{MULTI-SOURCE INFORMATION FUSION MODEL BASED ON ANFIS ALGORITHM FOR INTELLIGENT LIGHTING SYSTEM}

\section{A. Multi-Source Information Fusion Model for Intelligent} Lighting System

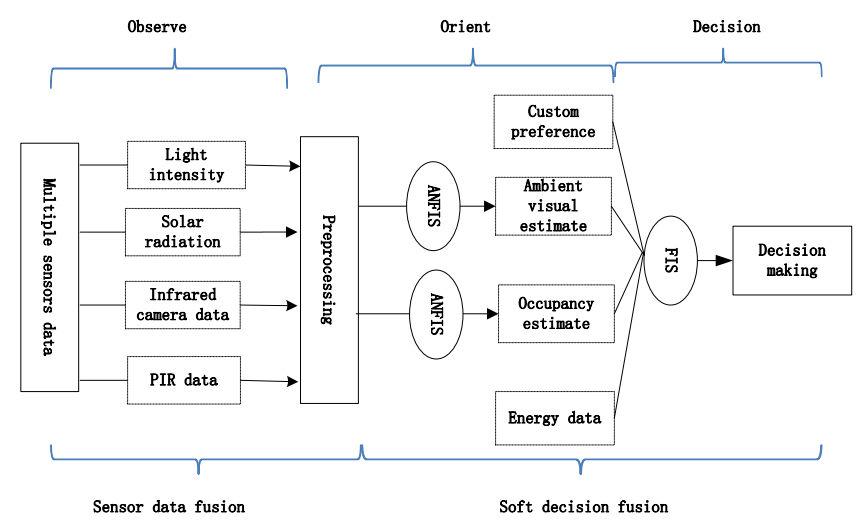

fig. 2. Multi-source information fusion model based on ANFIS algorithm

Ambient visual data, occupancy condition data and energy data, these three types of data obtained from the sensor network. Ambient visual data refer to building's environmental parameters such as the solar radiation, illumination intensity etc. Light intensity directly reflect the degree of indoor light and shade, it is the most important factor in the lighting control. We should make full use of 
natural light during the day, but it must be control of direct sunlight and radiation harmful rays. At night we could not take advantage of natural light, but it is necessary to control various of light pollution. So when we make the actual decision making, through a review of light intensity and solar radiation to control the percentage of the roller sunblind and the brightness of LED lamp.

Occupancy data usually include the number of occupants and presence/absence of occupants. The place with space larger, such as dining room, conferences room, classroom are divided into several area, through detecting the number of occupants in different area, to control turning on and turning off of the lamp, and data can be gained by PIR sensor.

Generally, energy data is dependent on energy supplies status such as the network condition and the price of electricity utilities. Other important inputs such as choice and convenience occupant behavior patterns are needed to realize the centralized-control user of building systems more effective. Comfort level measurement is always a problem worth studying. In the lighting system, visual comfort preferences are considered suitable for light and light color. Behavior patterns mainly refer to the user's lighting commonly used time, delay setting, and more information such as scene mode. For example when you work using the work mode, when you take a rest using the sleep mode, when you away from home using away-home mode.

The main objective of this work is decision making based on ambient visual level, occupancy condition, preferences of occupants, energy information. We can estimate the ambient visual level with light intensity and global solar radiation data based on adaptive neural-fuzzy inference system (ANFIS). Similarly, occupancy condition can evaluate with Passive infrared Detector (PIR) sensor data and infrared camera data. Preferences of occupants can be entered by user or learned by personal agent. Energy data comes from external input. In this fusion model, the sensor agents firstly can take sensor data fusion based on a data-level fusion algorithm to improve the detection accuracy. And then, local lighting agent makes a reliable ambient condition and an occupancy profile estimate using ANFIS algorithm. Finally, the system center makes decision combining multi-source information using a fuzzy inference system (FIS).

\section{B. Ambient Estimate and Pattern Learning using ANFIS Algorithm in Fusion Model}

ANFIS is a system that integrates neural networks and fuzzy inference system, which combines the learning ability of neural network and the ability to make decisions on fuzzy logic.

Let $x_{1}, x_{2}, x_{3} \ldots x_{m}$ indicates that $m$ input variable of system, each output value can be obtained from $n$ rules. Figure 3 is a typical architecture of ANFIS. This architecture shall be composed of five layers. Each layer consists of a number of nodes, where the number of nodes that perform the same task.

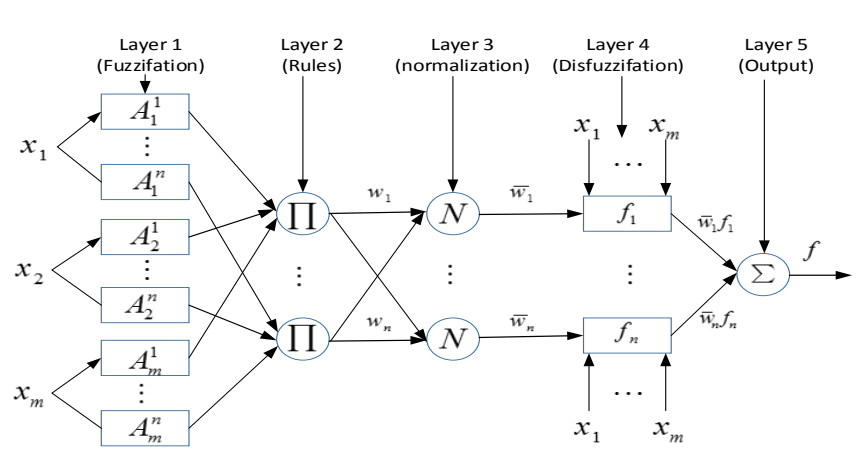

Fig. 3. The structure of ANFIS

Layer 1: Fuzzification layer, each node in this layer generates output in the form of its membership values based on fuzzy sets. The Node function may be either a Generalised Bell or Gaussian membership function. The outputs of this layer for different nodes are given by the following equation:

$$
O_{1, j}=\mu_{A I}^{k}\left(x_{i}\right)
$$

where $x_{i}$ is the input value $i=1,2, \cdots m ; k=1,2, \cdots n ; j=m \times n \cdot \mu_{A I}^{k}\left(x_{i}\right)$ is membership function, usually depicted bell-shaped function with a maximum value 1 and minimum value 0 :

$$
\mu_{A I}^{k}\left(x_{i}\right)=\frac{1}{1+\left(\frac{x-c_{i}^{k}}{a_{i}^{k}}\right)^{2 b_{i}^{k}}}
$$

where $\left\{a_{i}^{k}, b_{i}^{k}, c_{i}^{k}\right\}$ are premise parameters that need to adjust.

Layer 2: Rule layer. Layer two executes the fuzzy "AND" of the antecedent part of the fuzzy rules, the output of a node in this layer represents the firing strength of a fuzzy rule:

$$
O_{2, k}=w_{k}=\prod_{i=1}^{m} \mu_{A I}^{k}\left(x_{i}\right)
$$

where $k=1,2, \ldots n$.

Layer 3: Normalization layer, each node process and calculate the firing strength of each rule has been normalized.

$$
O_{3, k}=\bar{w}_{k}=\frac{w_{k}}{\sum_{i-1}^{n} w_{i}}
$$

where $\mathrm{n}$ is the total number of rules.

Layer 4: Defuzzification layer. Layer 4 is the defuzzification layer and is represented by $f_{x}$. Every node in 
this layer is an adaptive node. A defuzzification node calculates the weighted consequent value of a given rule and is simply the product of the normalized firing strength and the first order polynomial (for a first-order Sugeno model). The output of this layer is given by the following equation:

$O_{4, k}=\bar{w} f_{k}\left(d_{0}^{k}+d_{1}^{k} x_{1}+d_{2}^{k} x_{2}+\cdots+d_{m}^{k} x_{m}\right)$

Where $\left\{d_{0}^{k}, d_{1}^{k}, d_{2}^{k} \cdots d_{m}^{k}\right\}$ are conclusion parameters that need to learn.

Layer 5: Output layer. This layer is the output layer that composed of a single static node and denoted $f$. The output of the node is the accumulation of all the incoming signal. Therefore, the model output is generally expressed by the following equation:

$$
O_{5}=\sum_{i-1}^{n} \bar{w}_{k} f_{k}=\frac{\sum_{k=1}^{n} w_{k} f_{k}}{\sum_{k=1}^{n} w_{k}}
$$

The first layer run fuzzification process, each node in this layer generates output in the form of its membership values based on fuzzy sets. The Node function may be either a Generalised Bell or Gaussian membership function. Layer two executes the fuzzy "AND" of the antecedent part of the fuzzy rules, the output of a node in this layer represents the firing strength of a fuzzy rule. In the third layer, each node process and calculate the firing strength of each rule has been normalized. In layer four each node will calculate each of the previous rule with the model output. Last layer is the overall output of ANFIS by summing the output value of the fourth layer

ANFIS integrating neural network that is able to learn quickly and fuzzy system that is able to make decisions on uncertain information. To adjust the parameters of the premise and the conclusion, the ANFIS used back-propagation algorithm and a hybrid least squares algorithm, and will automatically generate the if-then rules. ANFIS is often used to shape the parameters of membership functions that is not certain, and also on the linear consequent parameters of fuzzy rules of the Takagi-Sugeno FIS. With this adjustment the fuzzy system able to do learning from the data that is modeled.

In our fusion model, we mainly use ANFIS to estimate occupancy level and learn model of human behavior.

\section{SIMULATION EXPERIMENTS}

In this section, we use android phone and personal computer to build multi-agent platform based on Jade agent, and use ANFIS to learn the position control of roller sunblind and brightness control of the LED lamp based on light intensity and solar radiation level.

\section{A. Simulation Platform.}

Reference [8] shows implementation of lightweight embedded agent based on android platform. Using it's principle, we develop a simple multi-agent platform between personal computer and android mobile phone. Software and hardware for simulation platform which are : a solar radiation meter, two android mobile phone, computer which was installed Jade Agent plug-ins, MATLAB simulation tool and Java development platform. Sensors agent was deployed on a android mobile phone as the agent to collect data from sensor.in order to simulated system sensor in the household system. MATLAB simulation tool on computer as a backstage analysis tool is used to simulate control center in smart home. Local agent was deployed on the computer, in order to receive the sensor data and saved those sensor data into local files. And personal agent was deployed on another mobile phone to received data from local agent and person.

\section{B. A Case Study using ANFIS for Information Fusion}

Multi sensors collect the actual environment of solar radiation, outdoor light intensity and indoor light intensity. We control the open ratio of roller blind and control the brightness of LED lamp respectively using ANFIS. In first ANFIS, we use the difference value between outdoor light intensity and indoor light intensity, solar radiation as input variables. The percentage of open ratio (range $0-1,0$ means fully closed and 1 means fully open) was the output variable. In second ANFIS, we use two input variables which are the percentage of open ratio, the difference value between indoor light intensity and user preference, and brightness of the LED lamp is the output of second ANFIS.

From $7.00 \mathrm{am}$ to $16.55 \mathrm{pm}$, we do the data collection every 5 minutes. there are 120 groups of indoor light intensity and solar radiation data totally. The outdoor light intensity is generally greater than 10000 according to our monitoring, so we assume that all of them are 11000; the time series diagram shown in figure 4 .

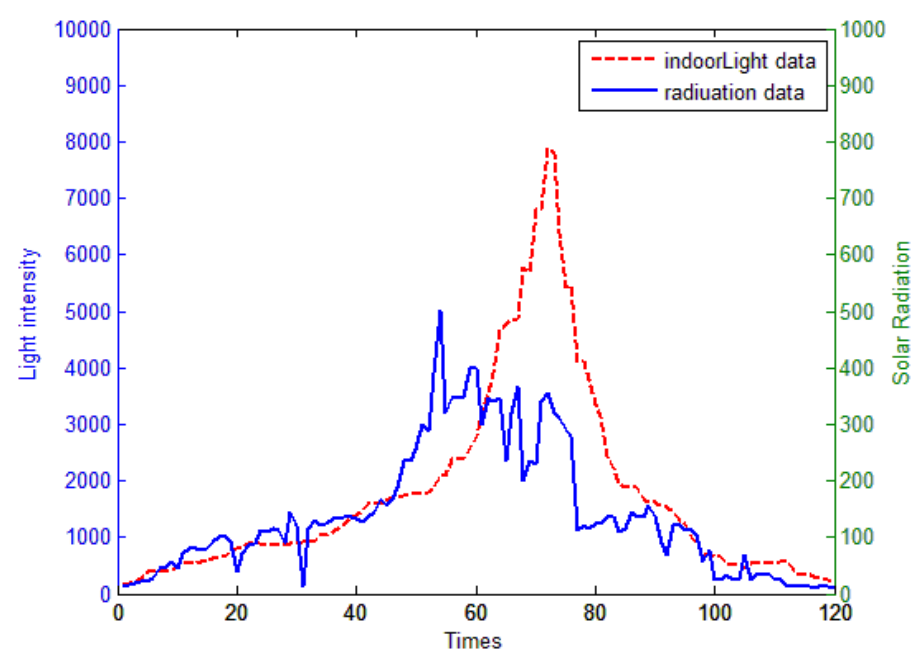

Fig. 4. Indoor light intensity and solar radiation time series diagram

In the first ANFIS model, we learn the percentage of sunblind openness. At first, we did the fuzzy classification of 
the input variables, the difference value between indoor light intensity and outdoor light intensity was classified as: positive big (PB), positive medium (PM) and positive zero (PZ), negative small (NS), negative medium (NM), negative big (NB). Solar radiation was classified as: radiation high $(\mathrm{RH})$, radiation medium $(\mathrm{RM})$ and radiation low(RL). According to human experience, we get the position of roller blind as the output variables. We set fifteen if-then reasoning rules, choose the bell-shaped function as the membership function. The data was divided into two groups which are training group(80 samples) and test group(40 samples). Firstly we did data normalization, after that, through the ANFIS reasoning algorithm toolbox in MATLAB simulation tool we did the simulation of natural lighting control. The structure of ANFIS in simulation experiment shows in figure 5 .

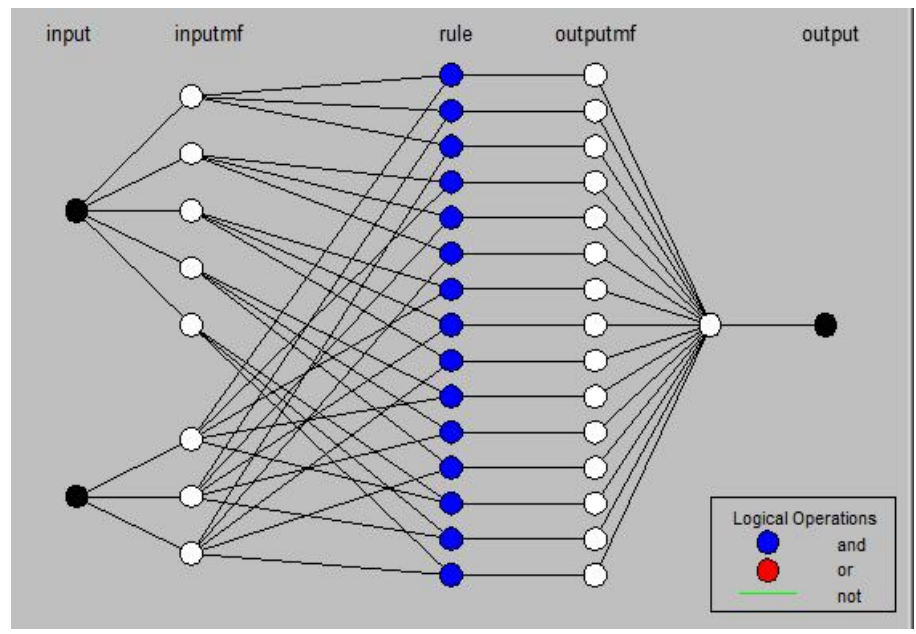

Fig 5 The structure of this ANFIS in simulation experiment

\section{CONCLUSIONS}

In this work, a multi-agent system is developed to control the intelligent light system based on multi-source information fusion. A sensor agent specially designed to obtain the multi-sensor data, and a personal agent is designed to enable the interactions between the occupants and the ambient environment by learning the behaviors of occupants. According to the case studies and simulation results, the proposed multi-agent system is capable of controlling the light effectively to satisfy occupant's demand, and the model based on ANFIS algorithm is very suitable for light control.
Moreover, it provides an open architecture in which agents can be easily configured, it also can be adapted to different home environments with various functionalities. The information fusion model also can extend to other smart home system. In the future work, we will improve the model, and extend the model to other smart home subsystems.

\section{REFERENCES}

[1] Wang Z, Wang L, Dounis A I, et al. Multi-agent control system with information fusion based comfort model for smart buildings[J]. Applied Energy, 2012, 99: 247-254.

[2] Yang R, Wang L. Development of multi-agent system for building energy and comfort management based on occupant behaviors[J]. Energy and Buildings, 2013, 56: 1-7.

[3] Ekwevugbe T, Brown N, Fan D. A design model for building occupancy detection using sensor fusion[C]//Digital Ecosystems Technologies (DEST), 2012 6th IEEE International Conference on. IEEE, 2012: 1-6.

[4] V.R. Budyal, S.S.Manvi.ANFIS and agent based bandwidth and delay aware anycast routing in mobile ad hoc networks.ELSEVIER, Journal of Network and Computer Applications 2014:140-151.

[5] Trobec Lah M, Zupančič B, Peternelj J, et al. Daylight illuminance control with fuzzy logic[J]. Solar Energy, 2006, 80(3): 307-321.

[6] Baetens R, Jelle B P, Gustavsen A. Properties, requirements and possibilities of smart windows for dynamic daylight and solar energy control in buildings: A state-of-the-art review[J]. Solar energy materials and solar cells, 2010, 94(2): 87-105

[7] Kristl Ž, Košir M, Trobec Lah M, et al. Fuzzy control system for thermal and visual comfort in building[J]. Renewable Energy, 2008, 33(4): 694-702.

[8] $\mathrm{Yu}$ Weihong. Intelligent light control system based on multi-agent and android sensor[J]. $\quad$ Computer\&Digital Engineering.2012.41(6):1009-1012.

[9] Yu Weihong,Chen Yan. Implementation of Lightweight Embedded Agent on Android Platform[J].Computer Engineering.2013.39(7):298-301.

[10] Raol J R. Multi-Sensor Data Fusion with MATLAB®[M]. CRC Press, 2009.

[11] Luansheng J, Chunxia L, Xiumei G, et al. The Design of Intelligent Lighting System in College Classroom[J]. Energy Procedia, 2012, 17: 90-95.

[12] Hassan M Y, Abdullah H, Rahman H A, et al. A review on lighting control technologies in commercial buildings, their performance and affecting factors[J]. Renewable and Sustainable Energy Reviews, 2014, 33: 268-279.

[13] Zhang J, Qiao G, Song G, et al. Group decision making based autonomous control system for street lighting[J]. Measurement, 2013, 46(1): 108-116 